\title{
Peinture navale et image de la nation en Angleterre (1660-1815)
}

Marine Painting and the Image of the English Nation (1660-1815)

\section{Michel Depeyre}

\section{CpenEdition}

\section{Journals}

\section{Édition électronique}

URL : http://journals.openedition.org/rfcb/1641

DOI : 10.4000/rfcb.1641

ISSN : 2429-4373

\section{Éditeur}

CRECIB - Centre de recherche et d'études en civilisation britannique

\section{Édition imprimée}

Date de publication : 1 janvier 2006

ISSN : 0248-9015

\section{Référence électronique}

Michel Depeyre, «Peinture navale et image de la nation en Angleterre (1660-1815) », Revue Française de Civilisation Britannique [En ligne], XIII-4 | 2006, mis en ligne le 01 janvier 2006, consulté le 19 avril 2019. URL : http://journals.openedition.org/rfcb/1641 ; DOI : 10.4000/rfcb.1641

Ce document a été généré automatiquement le 19 avril 2019

\section{(c) $(1)$}

Revue française de civilisation britannique est mis à disposition selon les termes de la licence Creative Commons Attribution - Pas d'Utilisation Commerciale - Pas de Modification 4.0 International. 


\title{
Peinture navale et image de la nation en Angleterre (1660-1815)
}

\author{
Marine Painting and the Image of the English Nation (1660-1815)
}

\author{
Michel Depeyre
}

1 Les historiens n'ont guère porté leur regard sur la peinture navale. Celle-ci est bien souvent utilisée comme illustration à destination des musées ou des ouvrages spécialisés, mais elle n'est pas étudiée pour elle-même. Elle est, en effet, rarement conçue comme une source à part entière de la recherche historique, tout comme le seraient un texte d'archive ou bien des mémoires de témoins de tel ou tel événement. Trop souvent l'historien estime que le tableau de bataille est œuvre de commande et, par là même, une source de mauvaise qualité qui répond aux attentes du commanditaire. À cela s'ajoute le rôle de l'imagination du créateur qui n'est donc pas censé être un témoin fidèle et digne de foi. Est-il d'ailleurs un témoin oculaire de la bataille décrite ? Négliger ou sous-estimer ces centaines de tableaux consiste, selon nous, à engendrer une grave lacune documentaire. En effet, non seulement cette peinture restitue une atmosphère dont nous n'avons que peu d'idée, mais elle est également un témoignage des représentations de l'époque des contemporains de l'événement, voire des thèmes idéologiques d'une société. Elle devient ainsi un véritable vecteur des images qu'un peuple et ses élites se font d'euxmêmes et qu'ils transmettent à la postérité.

2 L'importance quantitative de cette peinture est aussi fort révélatrice des enjeux présentés comme prioritaires par les États. Le petit nombre de scènes navales présentes dans la " galerie des batailles » édifiée à Versailles, en 1837, sous Louis-Philippe, est un indice de la dimension éminemment terrestre de la puissance politico-militaire de la France. Au contraire, l'Angleterre ne cesse de peindre ses succès sur mer, affichant la dimension maritime de sa puissance. Cette peinture sert un peu de miroir à la domination que le Royaume-Uni impose progressivement sur les océans entre la seconde moitié du XVII ${ }^{e}$ siècle et les débuts du XIX ${ }^{\mathrm{e}}$ siècle.

3 Comment pourrait-on, à l'usage de cette étude, définir et délimiter le corpus de cette source? Nous en exclurons les « marines » qui décrivent le monde maritime mais non la 
guerre sur mer. Il est question ici des tableaux de bataille qui constituent une partie du «grand genre » de la peinture d'histoire. Nous devons cependant associer les portraits des grands acteurs de ces combats, car ils puisent leur prestige dans les hauts faits navals. Ajoutons que l'iconographie qu'ils suscitent matérialise souvent certains thèmes patriotiques qu'il importe de prendre en compte. Comment le thème de la nation se manifeste-t-il dans la peinture navale anglaise ? Quand et comment ce genre est-il utilisé au service du patriotisme? Plus précisement encore, ce domaine particulier de la peinture est-il utilisé comme un moyen, entre les mains d'un groupe de pression, ou plusieurs, en vue de défendre des intérêts de natures différentes?

\section{Constitution de la puissance navale et de la peinture navale}

4 Avant de présenter la peinture navale, il est indispensable de situer notre problématique au sein d'un contexte plus large, celui du développement de la puissance maritime britannique. Quelques étapes capitales dans cette édification sont alors à souligner brièvement ${ }^{1}$. Henri VII est à l'origine de la marine anglaise, mais ce n'est qu'à la fin du $\mathrm{XVI}^{\mathrm{e}}$ siècle que les Anglais prennent en compte la dimension maritime de leur pays. La menace de l'Invincible armada de Philippe II d'Espagne, en 1588, est considérée, de ce point de vue, comme une étape essentielle de la prise de conscience anglaise. Raleigh, Drake, Hawkins contribuent beaucoup à ce processus. Dans les années 1617 ou 1618, John Selden donne à l'Angleterre un cadre juridique aux prétentions qu'elle pourrait avoir sur les mers. Le titre de l'ouvrage est indicatif: Mare clausum seu Dominium Maris. Contrairement à l'affirmation du Hollandais Grotius qui présentait la mer comme "res nullius ", la mer est, selon Selden, un espace que l'on peut s'approprier. Les Navigation Acts pris par Cromwell sont une concrétisation politique et économique de l'intégration par les dirigeants du rôle de la mer dans la vie de l'Angleterre. Dès lors, le choc avec des rivaux comme les Provinces-Unies et la France est inévitable. Dans la seconde moitié du $\mathrm{XVII}$ siècle, Charles II exprime de façon explicite la vocation maritime de l'Angleterre en affirmant que la frontière anglaise est située sur les côtes ennemies. Ainsi, la mer est envisagée comme un espace prolongeant naturellement le territoire insulaire de l'Angleterre. La nation anglaise a totalement assumé son rôle de puissance maritime à part entière. Le XVIII ${ }^{e}$ siècle lui permet d'afficher ses ambitions coloniales, notamment contre la France. De 1689 à 1815 se développe ainsi cette série de guerres que Jean Meyer réunit justement sous l'expression de "seconde guerre de cent ans ». Cet arrière-plan esquissé, la peinture navale anglaise traduit-elle ces moments du déploiement progressif de la puissance navale et maritime de l'Angleterre, puis du Royaume-Uni ?²

Si l'Espagne joue un rôle moteur dans la formation de la puissance maritime anglaise, les Provinces-Unies tiennent une place aussi importante. Les deux puissances protestantes deviennent vite des rivales sur les mers. Ce dangereux concurrent est cependant un maitre dans le domaine naval et - aussi - dans celui de la peinture. Les historiens de l'art constatent, en effet, que le genre de la peinture navale prend de l'importance à partir de la troisième guerre anglo-hollandaise et que l'apogée de ce type de peinture se place, pour l'Angleterre, dans la seconde moitié du siècle. Cela correspond exactement à l'époque des grandes victoires anglaises sur les Hollandais. 
6 Les Provinces-Unies ont donné naissance aux peintres de batailles les plus grands du moment, tels Willem van de Velde l'Ancien (1611-1693). Remarquons cependant que ni Vermeer ni Rembrandt ne se sont consacrés à ce genre. Van de Velde l'Ancien est le peintre officiel des grands combats hollandais ${ }^{3}$. Son influence est capitale pour tout le $\mathrm{XVIII}^{\mathrm{e}}$ siècle. Elle se fait sentir par l'intermédiaire de son fils, Willem van de Velde le Jeune (1633-1707), qui est le vrai fondateur de la peinture navale en Angleterre. En 1672, les deux artistes viennent s'établir en Angleterre. Van de Velde le Jeune peint alors des scènes de bataille vues du côté anglais, c'est-à-dire de l'ennemi. Il peint, par exemple, un épisode de la bataille anglo-hollandaise de Solebay qui eut lieu en 1672, lors de la troisième guerre anglo-hollandaise. La date du tableau est inconnue.

Quels sont les disciples anglais directs? Peter Monamy (1690-1748/49) a probablement travaillé dans l'atelier des van de Velde, Samuel Scott (1701/2-1772) et Dominic Serres (1722-1793) sont également à l'école des maîtres néerlandais ${ }^{4}$. À la théorie, Scott ajoute une pratique de la mer, puisqu'il fut embarqué à bord de bâtiments où il dessine des esquisses selon les habitudes et les techniques de van de Velde l'Ancien. L'influence des Hollandais peut être appréhendée par la présence de quatre compositions de van de Velde le Jeune dans le catalogue des œuvres possédées par Scott et vendues aux enchères après sa mort. Avec les Hollandais, la peinture navale est instituée comme un genre majeur dans les ateliers anglais.

$8 \mathrm{Au} \mathrm{XVIII}{ }^{\mathrm{e}}$ siècle, l'inspiration n'est plus hollandaise depuis la seconde moitié du siècle. Une école anglaise s'est constituée avec ses propres modèles, notamment Monamy. Au tout début du XIX siècle, le style est plus académique avec des hommes comme Hoppner, Benjamin West, Richard Westall, et avec l'arrivée de J.M.W. Turner (1775-1851), qui renouvelle le genre par des procédés qui s'éloignent des canons esthétiques hérités des Hollandais. La production des artistes anglais en scènes de bataille est imposante. Samuel Scott, par exemple, a peint durant sa carrière 29 tableaux de combats navals. Certains épisodes étant représentés plusieurs fois, 14 batailles sont effectivement peintes ${ }^{5}$. Tel est le cas de l'affaire du 20 juin 1743 contre le galion espagnol Nuestra Senora de Cavadonga, reprise trois fois par le peintre.

9 Il est intéressant de constater dans ce rapide inventaire des créateurs que plusieurs d'entre eux ne sont pas d'origine anglaise : les van de Velde sont hollandais, Serres vient de France, Philippe-Jacques de Loutherbourg arrive de Strasbourg. Cela ne les empêche pourtant pas de peindre les gloires navales britanniques. Jusqu'aux années 1680, Amsterdam est la "ville-monde " de "l'économie-monde atlantique » - pour reprendre les catégories de F. Braudel - issue des Grandes découvertes du XVI ${ }^{\mathrm{e}}$ siècle. Au tournant des XVII ${ }^{\mathrm{e}}$ et XVIII ${ }^{\mathrm{e}}$ siècles, Londres se substitue à la ville hollandaise. Du même coup, elle prend la place de premier centre de la peinture navale. L'arrivée de peintres étrangers est ainsi une sorte de marqueur mettant en évidence la prééminence maritime anglaise.

Quels sont maintenant les commanditaires de ces tableaux? Il est souvent difficile de le dire avec certitude. Les mécènes et les commanditaires sont pourtant un signe de l'intérêt porté à la peinture navale par les élites sociales et politiques de l'Angleterre. C'est ainsi que Samuel Scott, bien introduit dans la bonne société londonienne, reçoit des commandes d'hommes importants comme Horace Walpole. Quant à Dominic Serres, il devient en 1780 'appointed marine painter' de Georges III, ce qui prouve l'attention portée par la monarchie à ce type d'œuvre d'art. À ce titre, Serres répond à de nombreuses commandes ayant trait à la guerre d'Indépendance américaine. Après Aboukir, en 1798, le roi de Naples, Ferdinand, commande un tableau à Guy Head. Il représente Nelson blessé 
sur un fond de bataille où l'on distingue l'explosion de L'Orient. Le roi de Naples veut glorifier celui qui a sauvé son État des républicains. Il est désormais temps de considérer de plus près les thèmes évoqués dans cette peinture navale si riche. Un inventaire serait fastidieux, mais de grands thèmes peuvent néanmoins être distingués.

La puissance de la nation anglaise s'exprime en premier lieu par la flotte et les bâtiments qui la composent. Le vaisseau, The Man of War, est l'instrument principal de la puissance. Le regroupement en escadres permet de matérialiser le potentiel naval de l'Angleterre face à un ennemi souvent inférieur en nombre. Ces flottes assurent la sécurité des îles Britanniques, protègent les convois marchands et les colonies lointaines. Les représentations sur tableaux en sont innombrables. Vers 1736, Samuel Scott peint A FirstRate Shortening Sails. Nous avons sous les yeux un bâtiment de cent canons. Dominic Serres nous donne en 1778 The Royal George and Other Vessels'. Il s'agit, là encore, d'un vaisseau de premier rang de cent canons, un des plus puissants de la Navy. Une remarque s'impose devant les tableaux des maitres anglais : cette flotte anglaise ne comporte pas de galères. La présence d'un tel type d'unité sur un tableau anglais est un élément d'exotisme traduisant une localisation méditerranéenne de la scène. Tel est le cas d'un autre tableau de Scott intitulé An English Man of War Firing a Salute. Daté de 1785, la scène souligne l'écart de puissance existant entre l'Angleterre et les anciennes thalassocraties méditerranéennes, comme Venise ou Gênes, chez qui la galère est toujours une composante majeure de leur flotte ${ }^{7}$.

12 L'étude de certains détails des tableaux offre l'occasion d'identifier des éléments comme le système des pavillons et des signaux dont l'importance échappe fréquemment à l'historien. Les pavillons sont des outils indispensables pour le bon fonctionnement des flottes. Ils permettent de transmettre les ordres au sein de l'escadre. Ils sont aussi un moyen de reconnaissance entre les adversaires ${ }^{8}$. Arborer tel ou tel pavillon est porteur de sens pour un témoin oculaire. Il faut aussi dire que c'est afficher l'appartenance à un royaume ou à un pays. À ce titre, les couleurs sont un élément matérialisant la nation. Nous en voulons pour preuve la position centrale du pavillon de l'Union. Peter Monamy peint ainsi en 1709 le Queen Anne se mettant sur ancre avec la flotte. Au centre de la composition, le vaisseau amiral porte à son grand mât le pavillon de l'Union. À la poupe, il a monté le signal ordonnant à la flotte de se mettre sur ancre. 


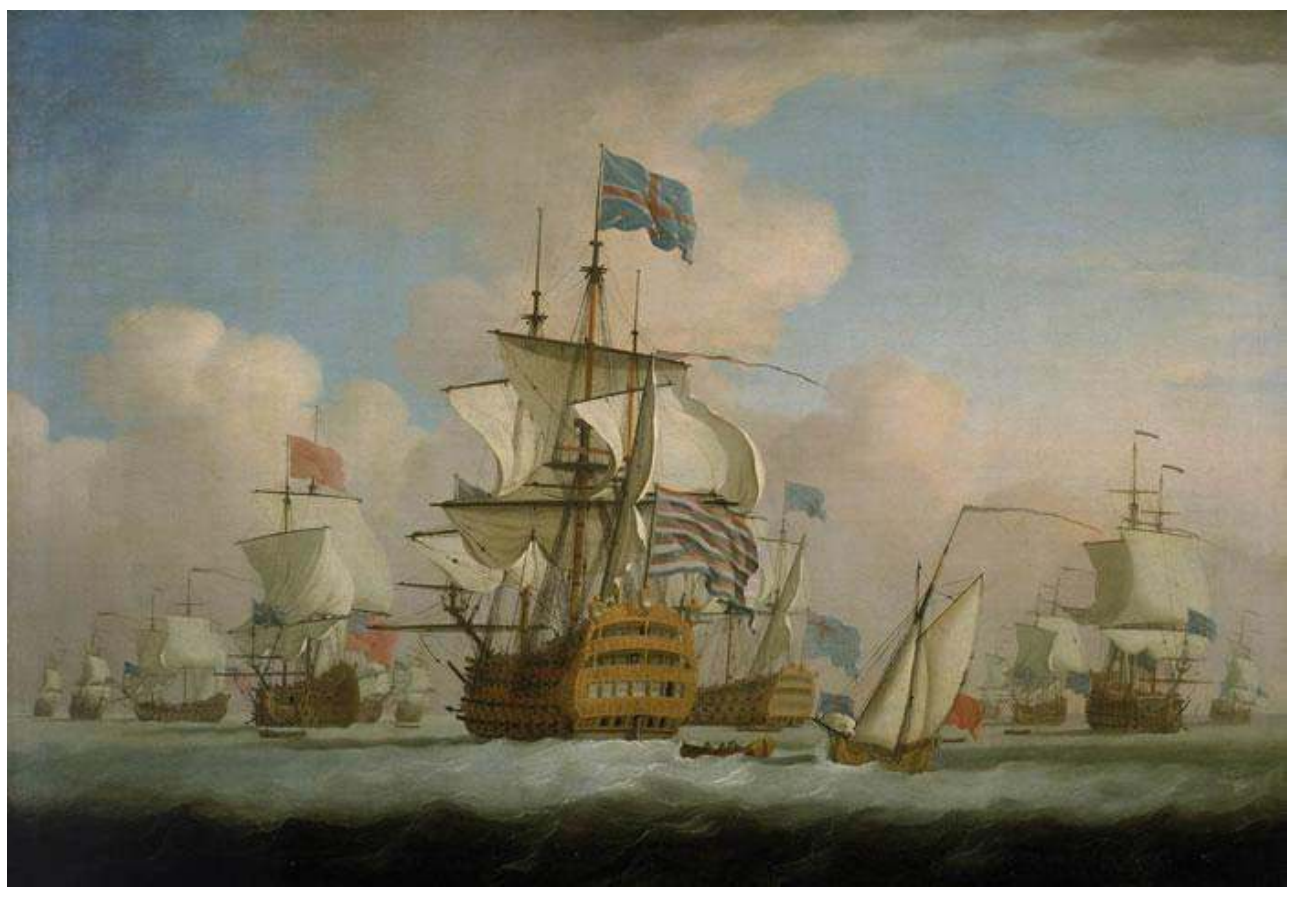

Sur le tableau de Samuel Scott représentant l'action d'Anson au Cap Finisterre, le 3 mai 1747 , on voit nettement trois pavillons sur le bâtiment placé au centre : le pavillon bleu sur le grand mât est la marque de l'amiral de la Blue division, Anson étant vice-amiral ; le pavillon rouge, blanc, bleu à rayures horizontales est un signal qui veut dire « chasser au sud-ouest ». Ce pavillon a été hissé vers les deux heures. Un troisième pavillon est placé au sommet du grand mât à 3 heures et signifie "attaquer sans ordre de bataille "* Aujourd'hui, ces pavillons ne nous parlent plus, alors qu'à l'époque le spectateur averti les comprenait. Taches multicolores, les signaux sont également le prétexte pour le peintre d'éclairer un tableau où les tonalités sombres l'emportent le plus souvent au cœur des canonnades. Le combat est, en effet, l'élément primordial de la peinture navale. Les opérations de la Royal Navy sont mises en scène dans toute leur diversité. À côté des batailles en lignes de file, selon le modèle tactique du temps, nous avons aussi des opérations de blocus, des manœuvres d'abordage. Les stratégies britanniques se révèlent de cette façon à l'historien. Lorsqu'à une date inconnue, Thomas Buttersworth décrit le blocus imposé par Nelson à Cadix, après la bataille de Saint-Vincent, durant le mois de juin 1797, il ne nous offre pas une page épique, et pourtant il pointe une des stratégies privilégiées par les Britanniques depuis la guerre de Sept ans, le blocus.

À une époque où les combats sont de plus en plus éloignés des îles Britanniques, la Royal Navy se donne à voir dans tout son éclat, légitimant du même coup sa fonction et les colossaux budgets qu'elle ne cesse de réclamer. Certains chiffres sont éloquents. L'Angleterre possède en 1689 cent vaisseaux de ligne, sans compter les frégates; en 1739, elle est passée à 124 vaisseaux; elle est retombée à 90 en 1779, pour remonter à 195 en 1790 et finir à 214 en $1815^{10}$. Il est aisé de constater l'effort prodigieux engagé à partir des années 1780. Cela impose des financements colossaux. La monarchie anglaise utilise les victoires de sa marine mais cette dernière se sert aussi de cette publicité pour défendre ses propres intérêts. Il convient, en effet, de ne pas omettre que les crédits sont alloués 
par le Parlement et qu'il faut régulièrement peser sur le Cabinet ou sur les hommes politiques.

15 La peinture navale nous livre donc une série de descriptions de la bataille. La guerre est mise en scène sous des couleurs qui cachent le plus souvent l'horreur des combats. Nous pouvons même aller plus loin. À partir des années 1660, l'Angleterre voit apparaitre une véritable esthétique de la bataille navale, avec ses peintres attitrés, ses canons picturaux, ses thèmes de prédilection. Parallèlement, la peinture navale, plus que le simple épisode relaté sur la toile, fixe avant tout la mémoire de l'événement concerné. Il se constitue ainsi une histoire nationale ponctuée de hauts faits. La nation se fonde largement sur cette mémoire qui est relayée par d'autres modes d'expression, arts ou écrits.

\section{Nation et valeurs anglaises}

L'esthétique de la bataille sur mer n'est donc pas désintéressée. Écrire cela ne saurait pourtant suffire. En effet, un contenu politique se dessine progressivement sous nos yeux. Nous assistons à la montée en puissance, parfois saccadée, de la «thalassocratie triomphante $»^{11}$ qui, après 1815 , et pour longtemps, n'a plus de rival dangereux. Dans ce contexte, comment donner vie à des idées ou à des principes? La musique permet de galvaniser des énergies, la peinture tient un autre rôle. En effet, dans ce vaste "musée imaginaire ", la peinture navale est également destinée à porter les valeurs réputées anglaises, comme le courage ou l'opiniâtreté. Cet éloge de l'âme britannique est déjà présent dans certains ouvrages techniques ou politiques, mais la peinture les montre au regard et les met en scène dans l'action. La force du message en est d'autant plus grande. $\mathrm{Au} \mathrm{XVIII}{ }^{e}$ siècle, les journaux relatent les événements, mais la peinture touche un plus grand nombre de personnes. Les peintres ont la possibilité de figer un moment remarquable lors d'un combat. Ils sélectionnent ainsi les instants décisifs (abordage, manœuvre hardie, canonnade...). Dans le traitement de ces épisodes de guerre, deux cas de figure peuvent se présenter à nous.

Dans le premier, il est question de retenir l'action individuelle d'un simple combattant ou d'un officier. Le courage, le sacrifice de tel ou tel marin est alors mis en exergue et offert à l'admiration collective. Dans le second cas, l'action cette fois-ci collective est privilégiée par le peintre. Nous savons que la littérature est un des moyens principaux pour mettre en exergue les vertus d'un groupe humain. Sous la Restauration, le poète John Dryden met en scène le courage anglais dans Annus Mirabilis, texte publié en 1666. Dans ce long poème de 304 quatrains il décrit le courage de son peuple face à trois catastrophes: la peste, le Grand incendie de Londres et la défaite de la bataille des Quatre jours (11-14 juin 1666) lors de la seconde guerre contre le concurrent du moment, les Provinces-Unies. La description des campagnes navales représente 208 quatrains, soit la plus grande partie du poèm $\mathrm{e}^{12}$. Un tel exemple surprend si nous le comparons avec le vide presque total dans la littérature française des $\mathrm{XVII}^{\mathrm{e}}$ et $\mathrm{XVIII}{ }^{\mathrm{e}}$ siècles en ce qui concerne les descriptions de combats à la mer. Dryden, malgré la défaite, retient la résistance de son peuple face à l'adversité et contre l'ennemi. Il appartient cependant au Hollandais Abraham Storck de peindre la victoire de l'amiral Michel De Ruyter. Une école britannique de peinture navale étant désormais largement reconnue, un artiste anglais peut maintenant décrire le courage de ses concitoyens. L'Histoire a, en revanche, gardé en mémoire des contreexemples. C'est ainsi que le 14 mars 1757, à Portsmouth, sur le pont de son vaisseau amiral le Monarch, l'amiral John Byng est fusillé à la suite d'un procès devant une cour 
martiale. Ce marin de qualité est jugé responsable de la perte de la base navale de Minorque l'année précédente, laissée aux Français. L'événement stigmatise le manque de combativité d'un officier général. Un peintre anonyme de l'école anglaise du XVIII siècle a peint à une date inconnue The Capture of Admiral Byng.

À côté des vertus du combattant, la science navale de l'Angleterre est également un objet de louanges. Les mouvements ordonnés d'une flotte soulignent l'art de la tactique pratiqué par la Navy. L'Anglais est présenté comme le meilleur marin au monde par le tacticien John Clerk of Eldin dans son Essay on Naval Tactics, dont le premier volume est publié en $1790^{13}$. Malgré des origines écossaises, l'auteur est d'un ardent patriotisme anglais, montrant ici encore le pouvoir d'attraction de la puissance navale du Royaume$\mathrm{Uni}^{14}$. Revendiquer et proclamer des valeurs et des principes ne suffit pas. La nation se constitue aussi en s'affirmant contre l'Autre, l'ennemi. Au XVII ${ }^{\mathrm{e}}$ siècle, celui-ci est principalement hollandais; de la fin du XVII ${ }^{e}$ siècle au début du XIX ${ }^{e}$ siècle, il est essentiellement français. Nous avons affaire à des concurrences politiques, économiques et coloniales, l'enjeu géostratégique étant la domination sur "l'économie-monde atlantique ». Nombre de peintures portent sur le gigantesque conflit franco-anglais qui s'étend de 1690 à 1815. Des deux côtés de la Manche, un sentiment national s'exacerbe lors des phases actives de guerre ${ }^{15}$. Chaque victoire donne lieu à un tableau. Jusqu'en 1783, nous demeurons pourtant dans un style de guerre classique. Samuel Scott et Dominic Serres en fournissent de nombreux exemples. Les règles canoniques du combat sur mer codifiées dans la seconde moitié du XVII siècle sont le plus fréquemment respectées.

19 La période des French Wars, entre 1793 et 1815, est cependant marquée d'une teinte idéologique nouvelle. Nous passons de la guerre des rois à la guerre des idéologies. Les belligérants sont entrés dans la première étape de la guerre totale ${ }^{16}$. Les ennemis en présence cherchent désormais à anéantir l'adversaire et son idéologie, révolutionnaire ou conservatrice. Les peintres perçoivent cette tonalité nouvelle. Le tableau de Philip James de Loutherbourg (1740-1812) qui représente le combat du 13 prairial (1 ${ }^{\text {er }}$ juin 1794) entre le royaume de Grande-Bretagne et la République française en fournit ainsi un bel exemple. Le désordre des flottes est extrême, le Queen Charlotte passe sous le vent du Montagu mais ne réussit pas à atteindre le vaisseau amiral français. L'ordre tactique a totalement disparu, le combat est acharné et sans ordre. Le combat est à outrance. La Révolution glorifie la résistance désespérée du célèbre Vengeur. Cela devient un des thèmes de prédilection de la propagande iconographique de la Convention. Les peintres britanniques ont remarquablement traduit ces évolutions. La peinture enregistre de manière explicite les changements qui s'opèrent dans les tactiques navales mises en œuvre ${ }^{17}$. On est pourtant en droit de s'interroger sur la prise de conscience qui pourrait naître chez les dirigeants comme William Pitt. Les peintres mémorisent une transformation de l'esprit de la guerre, mais celle-ci est-elle bien comprise? Nous savons, en effet, que ce n'est qu'en 1795 que le Premier ministre Pitt et ses collaborateurs découvrent que la guerre ne peut plus être conduite comme auparavant. Avec les campagnes de la Révolution et de l'Empire, la guerre change de nature et cela est bien traduit sur certains tableaux. Face à la "Grande Nation", le Royaume-Uni, et plus spécifiquement l'Angleterre, affirme son opposition irréductible. Le courage de l'un ou de l'autre devient exemple pour tous. Les historiens de l'Antiquité, comme Plutarque, avaient déjà mis en exergue l'importance des exempla. Chacun est un fragment de la nation qui lutte pour la survie de tous. 


\section{Le héros naval, incarnation de la nation}

Glorious Revolution, ou sur la mémoire des grandes confrontations avec l'ennemi, comme la bataille de Trafalgar, mais elle doit aussi s'incarner dans de fortes personnalités qui sont autant d'exempla qui marquent la mémoire collective. Les historiens de cette époque s'attachent d'ailleurs à la description des grandes figures et de leurs actions d'éclat. Cela se ressent dans The Naval History of England in all of its branches... de Thomas Lediard ${ }^{18}$, mais également dans The Naval Chronicle ${ }^{19}$, publiée entre 1793 et 1799. Ce ne sont pas les actions spectaculaires des souverains mais la bravoure d'une marine. Il ne faut cependant pas être dupe de cet unanimisme. Il est, en effet, obtenu par une discipline inflexible. Les mutineries sur la Nore, en 1797, prouvent que la situation politique et sociale du royaume est très tendue. William Pitt conduit la nation mais celle-ci n'offre pas l'unité sans faille que l'on se plait à décrire. Les esprits sont fatigués par une guerre interminable et qui multiplie les charges fiscales. Comble de tout, les révoltes en Irlande menacent de prendre le Royaume-Uni sur son flanc occidental ${ }^{20}$. La peinture navale est, notamment à la fin du XVIII siècle, l'outil idoine pour concentrer l'attention des sujets sur l'objectif à atteindre, la victoire anglaise quoi qu'il en coûte.

galeries des demeures anglaises abondent en portraits d'ancêtres plus ou moins glorieux. Ils matérialisent la mémoire et la continuité des lignages nobles. Ce genre de peintures est aussi bien commandé par l'État que par des particuliers. Grandes dynasties et pouvoir politique commémorent ainsi les figures de proue de leur histoire. Les marins ne font pas exception. Parmi les peintres, nous retrouvons les noms des plus grands portraitistes de l'époque, tel Joshua Reynolds qui, en 1786, peint l'amiral Keppel. En 1789, il fait un portrait en pied représentant George Brydges, First Lord Rodney. Le portrait en pied autorise alors, souvent en arrière-plan, le rappel des victoires. C'est notamment le cas des amiraux comme George Byng, vicomte de Torrington, représenté en grand habit de lord, après sa mort en 1733, par Jeremiah Davison. En arrière-plan à droite du tableau, Davison a peint la grande victoire du défunt, au large du cap Passaro, en Sicile, victoire remportée contre les Espagnols en 1718.

La place tenue par Horace Nelson est néanmoins sans équivalent dans les deux siècles qui précèdent. Très nombreuses sont les représentations iconographiques du héros, et ce, sur tous les supports possibles (papier, toile mais aussi tissu, céramique, porcelaine...). Les portraits officiels sont plus particulièrement intéressants à analyser. Certes, ils fournissent quelques informations sur la personnalité du marin, mais surtout, nous pouvons appréhender la transmutation de l'homme en héros de l'Angleterre. Nelson fait figure de sauveur du royaume face à la France révolutionnaire et impériale. Le sentiment anti-français s'incarne dans ce combattant qui refuse de parler le français et qui a porté un coup sérieux aux prétentions ennemies. Nelson représente le courage sous tous ses aspects. En 1806, Richard Westall le peint ainsi en train de se lancer à l'abordage, en 1777, d'un bâtiment de commerce américain. Il est probable que le jeune Nelson n'a pas tenu à bord du Lowestoffe le rôle qui lui est ici attribué, mais cela importe peu au regard de la postérité. L'adversaire est alors américain. Le même phénomène est repérable pour les événements des décennies suivantes. Le défenseur de l'Angleterre contre Napoléon se voit alors paré de tous les mérites et devient un véritable paradigme pour les jeunes officiers et l'ensemble des Britanniques. 

le sacrifice de l'amiral. En effet, Neptune - sur la gauche du tableau - offre le corps du défunt à Britannia. Nous voyons bien dans ce cas comment Britannia n'offre plus le défunt mais le reçoit dans une attitude déifiée. Un ange a écrit sur un rouleau de parchemin le célèbre signal de l'amiral transmis à toute la flotte, un peu avant le début de la bataille de Trafalgar : 'England expects that every man will do his duty'. Nelson incarne le devoir au service de la patrie mais il est surtout celui qui galvanise ses équipages par son courage personnel. Lors des funérailles de Nelson, toute l'Angleterre a communié dans le culte du héros. Depuis Greenwich, sur un bâtiment de la Navy, la dépouille a remonté la Tamise sous le regard des populations. Les œuvres d'art qui décrivent cette héroïsation portent le souvenir de ce moment d'unité nationale.

einture navale anglaise enregistre les hauts faits et héroïse les marins victorieux. Elle fait cependant plus encore puisqu'elle prend en charge les grands symboles de la mémoire nationale. Il est ainsi possible d'écrire qu'elle est un de ces « lieux de mémoire » conceptualisés par Pierre Nora ${ }^{21}$. Selon ce dernier, une nation se constitue tout au long de son histoire un patrimoine mémoriel fondateur, dans lequel elle puise régulièrement pour commémorer sa naissance, son existence et les grands faits qui jalonnent son histoire. Ces « lieux » peuvent être matériels, comme le Panthéon à Paris, ou immatériels, comme La Marseillaise. Avec ces "lieux de mémoire » sont véhiculés des principes et des valeurs reconnus du plus grand nombre. Une reconnaissance consensuelle est le critère nécessaire pour individualiser un de ces « lieux de mémoire ». 
Un tel concept s'applique particulièrement bien à la peinture navale anglaise. Certes, l'ensemble de ces œuvres n'est pas vu ni connu d'une large proportion de la population anglaise, mais il en va de même d'une très grande partie de la production artistique et littéraire du temps. Les élites sociales et politiques sont prioritairement concernées par ces modes d'expression. Outre que les élites voient dans ces tableaux une glorification de leurs mérites, ces tableaux matérialisent également la victoire d'un système politique sur un autre. Le modèle anglais fruit de la Glorious Revolution l'emporte sur celui de la monarchie absolue française, puis sur celui de la Révolution française.

Dans ce contexte politico-culturel, le Victory, vaisseau amiral de Nelson, devient rapidement un «lieu de mémoire» dans toutes les acceptions possibles. Les tableaux permettent alors de répandre dans tout le royaume le souvenir de la relique soigneusement entretenue, mais aussi la mémoire du héros divinisé. Il faut cependant aller plus loin dans l'analyse. La peinture navale, apprise des Hollandais, est désormais devenue une spécialité britannique. En enregistrant l'ensemble des gloires navales, ce genre pictural à part entière acquiert pleinement la dimension d'un " lieu de mémoire ». Elle ne sert pas à illustrer les événements décrits, elle porte entièrement les aspirations et les grandeurs d'une nation qui n'a rien pourtant du monolithisme revendiqué de la "Grande Nation", c'est-à-dire la nation française. Au Royaume-Uni, les héros sont anglais mais aussi gallois ou écossais. La peinture navale fusionne le sacrifice de tous ces hommes dans la défense des îles Britanniques. Il est clair qu'une telle revendication par les élites du consensus national autour de la dynastie régnante oblitère la réalité. Pitt réprime durement la révolte irlandaise et les mutineries de la Nore en $1797^{22}$.

Ainsi, tout au long de notre période, navires et combats, tout en restant primordiaux, laissent la place à la figure du héros. Le combat passe progressivement du premier plan à l'arrière-fond. Les peintres continuent de composer de vastes tableaux mettant en scène le choc entre ennemis mais l'essentiel n'est plus là. La figure de Nelson fait plus pour la cause nationale que la représentation de la bataille de Trafalgar avec toute l'exactitude historique voulue.

1 L'historien bénéficie, avec la peinture navale, d'une source abondante et dont les enseignements sont multiples et féconds. Dans le cas plus précis de l'Angleterre, nous nous trouvons en présence d'une mine presque inexploitée jusqu'à présent. Outre la restitution d'une atmosphère qui est immédiatement rendue présente au spectateur par nombre de détails évocateurs (lumière, fumée, désordre...), l'observateur découvre les éléments d'une idéologie de la nation, avec ses symboles (comme les pavillons), ses victoires et ses héros. Un véritable discours pictural donne un sens à cette immense production.

Faire un tel constat ne suffit pourtant pas, car une approche chronologique fait défaut. En effet, sur la longue durée, une tendance se dessine assez nettement : le thème patriotique prend une importance grandissante, tout particulièrement avec les guerres de la Révolution et de l'Empire. Pavillons, attributs divers (trident de Neptune, allégories), exaltation des figures héroïques deviennent de plus en plus nombreuses. Ces dernières permettent de renforcer et de donner chair à la conscience nationale parfois déstabilisée par la propagande révolutionnaire. Le discours acquiert une efficacité toujours plus grande. De moyen d'expression de la puissance anglaise sur les océans, la peinture navale devient un outil à part entière de la propagande patriotique. Il appartient cependant aux milieux navalistes (notamment avec la Blue Water School) et coloniaux du XIX ${ }^{e}$ siècle d'en amplifier la portée auprès des populations et de susciter un authentique nationalisme. De 
ce point de vue, l'iconographie nelsonienne, « lieu de mémoire » dans toute l'acception du terme, est un prodigieux moyen pour galvaniser les énergies britanniques.

\section{NOTES}

1. Pour des analyses plus poussées voir Michael LEWIS, The Navy of Britain, London: George Allen \& Unwin, 1949, $660 \mathrm{p}$.

2. Nous retiendrons pour ces deux qualificatifs dans le sens retenu par les Anglais : " maritime » recouvre l'ensemble des activités en rapport avec la mer (commerce, guerre, explorations...), alors que «naval » ne s'applique qu'au domaine restreint à la guerre sur mer. Le premier terme est donc plus large que le second.

3. Ellis WATERHOUSE, Painting in Britain, 1530-1790, The Pelican History of Art, London: Penguin, $3^{\mathrm{e}}$ éd. 1969 , p. 103.

4. C. BENEZIT, Dictionnaire des peintres, sculpteurs, dessinateurs et graveurs, Paris : Gründ, nouvelle édition 1966, t. VII, 684 p. Serres est d'origine française et a été marin dans la flotte de commerce. 5. Voir R. KINGZETT, A Catalogue of the Works of Samuel Scott, London: Walpole Society, vol. 48, 1982.

6. Ce vaisseau chavire et sombre le 28 août 1782, faisant ainsi un très grand nombre de victimes ; voir Brian LAVERY, The Ship of the Line, London: Conway, 1983, 2 vol., t. I, pp. 88-89.

7. En France, le corps des galères disparaît en 1765.

8. Brian TUNSTALL, Naval Warefare in the Age of Sail, London: Conway, 1990, p. 127 ; voir table des signaux de l'amiral Hawke durant la guerre de Sept ans.

9. Elizabeth EINBERG \& Judy EGERTON, The Age of Hogarth : British Painters born 1675-1709, London: Tate Gallery, 1988, p. 185.

10. Paul KENNEDY, Naissance et déclin des grandes puissances, trad. fr., Paris : Payot, 1988, p. 134.

11. Philippe CHASSAIGNE, La Grande-Bretagne et le monde de 1815 à nos jours, Paris : A. Colin, 2001, p. 5.

12. Voir Pierre LEGOUIS, Dryden : Poèmes choisis, Paris : Aubier, 1946, 443 p.

13. Michel DEPEYRE, Tactiques et stratégies de la France et du Royaume-Uni, de 1690 à 1815, Paris: Economica, 1998, p. 165.

14. Le père de John est un des rédacteurs de l'Acte d'Union de 1707 entre l'Angleterre et l'Écosse.

15. Jean GUIFFAN, Histoire de l'anglophobie en France, Paris : Terre de brume, 2004, pp. 73 \& seq.

16. Jean-Yves GUIOMAR, L'invention de la guerre totale, XVIII ${ }^{e}-X X^{e}$ siècles, Paris : Le Félin, 2004, 331 p., pp. 87 \& seq.

17. Michel DEPEYRE, Entre vent et eau: Un siècle d'hésitations tactiques et stratégiques, 1790-1815, Paris : Economica-CFHM, 2003.

18. Thomas LEDIARD, The Naval History of England in all of its Branches from the Norman Campaign in the Year 1066 to the Conclusion of 1734, London: Wilcar, 1735, 394 p.

19. The Naval Chronicle, London: Joyer \& Gall Print. Il s'agit d'une chronique qui rapporte régulièrement les opérations navales.

20. Voir notamment Jules DESCHAMPS, Les îles Britanniques et la Révolution française (1789-1803), Paris : La Renaissance du livre, 1949, 244 p., pp. 87 \& seq.

21. Pierre NORA, Les lieux de mémoire, Paris : Gallimard, 1984, t. I, p. xxxiv.

22. Jules DECHAMPS, Les îles Britanniques et la Révolution française, pp. 83 \& seq. 


\section{RÉSUMÉS}

La peinture navale devrait être davantage utilisée comme source d'information par les historiens. Cet article sera surtout consacré aux «marines " décrivant la guerre sur mer, les tableaux de bataille, utilisés au service du patriotisme national. C'est à la fin du XVII ${ }^{\mathrm{e}}$ siècle que les Anglais prennent conscience de leur puissance maritime face à deux ennemis principaux, les Provinces Unies et la France, deux nations puissantes sur mer et riches en peintures navales. Willem van de Velde l'Ancien et Le Jeune influencent Samuel Scott, leur disciple anglais, auteur d'un grand nombre de «marines ». Parmi les thèmes majeurs de ces tableaux, le Vaisseau de Guerre, la Flotte tout entière avec ses pavillons, sa tactique militaire, sa puissance, et bien sûr le combat proprement dit. Ces batailles expriment les valeurs fondamentales de la nation anglaise : le courage, l'opiniâtreté, le sacrifice des marins pour la patrie - le tout illustré dans de gigantesques conflits, notamment les guerres franco-anglaises (1793-1815). Le héros par excellence qui incarne ces vertus, c'est Nelson, forte personnalité destinée à marquer la mémoire collective. Les nombreux tableaux qui le mettent en scène, notamment à Trafalgar insistent sur son corps mutilé, offrande à sa mère patrie pour la défense de sa grandeur et de sa noblesse.

Marine painting should be better used by historians as a source of information. This article is mainly devoted to the pictures illustrating battles at sea, as illustrations of national patriotism. It was at the end of the $17^{\text {th }}$ century that the English became conscious of their own power at sea, faced with two of their main enemies, France and the Netherlands, two powerful maritime powers, also rich in marine paintings. Willem van de Velde Senior and Junior were certainly the models of the English painter Samuel Scott, the author of a vast quantity of marine paintings. Among the subject matters of such works, there were the Man of War, the whole Fleet with its flags, its tactics and power, its symbolism, and of course the battle itself. Such battles at sea were meant to embody the basic values of the English nation : courage and stubbornness, the willing sacrifice of seamen for their mother country. Such were the themes of all marine paintings during the gigantic Anglo-French wars at the time of the French Revolution and the Napoleonic Wars (1793-1815). The supreme hero was of course Nelson, the symbol of all values to be stamped in the collective memory. Many pictures were devoted to him, whose mutilated body, at Trafalgar, was represented as the ultimate sacrifice to his mother country, for the preservation of its greatness and nobility.

\section{AUTEUR}

\section{MICHEL DEPEYRE}

Université Jean-Monnet (Saint-Étienne) 